\title{
A SYSTEM OF AXIOMATIC SET THEORY - PART VII ${ }^{84}$
}

\section{PAUL BERNAYS}

18. Further models for proofs of independence. The reader of Part VI will have noticed that among the set-theoretic models considered there some models were missing which were announced in Part II for certain proofs of independence. These models will be supplied now.

Mainly two models have to be constructed: one with the property that there exists a set which is its own only element, and another in which the axioms I-III and VII, but not Va, are satisfied. In either case we need not satisfy the axiom of infinity. Thereby it becomes possible to set up the models on the basis of only I-III, and either VII or Va, a basis from which number theory can be obtained as we saw in Part II.

On both these bases the $\Pi_{0}$-system of Part VI, which satisfies the axioms I-V and VII, but not VI, can be constructed, as we stated there. An isomorphic model can also be obtained on that basis, by first setting up number theory as in Part II, and then proceeding as Ackermann did. ${ }^{85}$

Let us recall the main points of this procedure.

For the sake of clarity in the discussion of this and the subsequent models, it will be necessary to distinguish precisely between the concepts which are relative to the basic set-theoretic system, and those which are relative to the model to be defined.

Unless otherwise stated, the terms are to refer to the basic system, so that in particular the term "natural number" (or simply "number", when no ambiguity seems possible) means "finite ordinal of the basic system". Furthermore in order to indicate the rôle of the natural numbers as constituting the sets of the model we speak of them as "new sets".

We now proceed to the description of Ackermann's number-theoretic model, which we call briefly the model $\mathfrak{M}$. The sets of the model $\mathfrak{M}$ are the natural numbers, and the element relation in $\mathfrak{M}$, which we denote by " $m \tilde{\boldsymbol{\epsilon}} n$ ", is defined to mean that the greatest natural number $k$ such that $k \cdot 2^{m} \leq n$ is odd. (It may be recalled that the relation $r<s$ between natural numbers $r, s$ in our system is simply $r \in s$.) The condition amounts to requiring that $2^{m}$ occurs as a term in the expansion of $n$ as a sum of different powers of 2. Classes in $\mathfrak{M}$ are number classes, and the relation $m \eta A$ is the

Received March 30, 1953.

${ }^{84}$ Parts I-VI appeared in this Journal, vol. 2 (1937), pp. 65-77; vol. 6 (1941), pp. 1-17; vol. 7 (1942), pp. 65-89, 133-145; vol. 8 (1943), pp. 89-106; vol. 13 (1948), pp. 65-79.

${ }^{85} \mathrm{~W}$. Ackermann, Die Widerspruchsfreiheit der allgemeinen Mengenlehre, Math. Ann. vol. 114 (1937), pp. 305-315. 
same as in the basic system. Also the identity relation between sets and between classes remains unchanged.

For verifying that the model so defined satisfies all the axioms $I-V$ and VII, it will suffice to give the main ideas.

That the extensionality axiom holds is immediate.

As to the axioms II we have: The number 0 is the null set. The augmentation of a new set $m$ by a further element $n$ consists in the operation $m+2^{n}$. An ordered pair $\langle m, n\rangle$ of new sets is either the number $2^{2^{m}}$ in case $m=n$, or $2^{2^{m}}+2^{2^{m}+2^{n}}$ otherwise.

For the verification of the axioms III we can rely on the following circumstances. There exists the class of natural numbers. The relation $\tilde{\boldsymbol{\epsilon}}$ can be formulated by a constitutive expression, ${ }^{86}$ likewise the ternary relation $\left(a=b \& c=2^{2^{a}}\right) \mathbf{v}\left(a \neq b \& c=2^{2^{a}}+2^{2^{a}+2^{b}}\right)$. From the latter in particular it follows that, for every class of pairs (in the old sense) of new sets, there exists the class of the corresponding pairs in the model, and inversely. Likewise it is to be noted that for a class of pairs in the model the domain and also the converse domain is (by the definition of the "member" of a new pair) the same as for the class of corresponding pairs in the basic system. - As a consequence of the holding of the axioms III in $\mathfrak{M}$ the class theorem is available for $\mathfrak{M}$.

The validity of the axiom IV in $\mathfrak{M}$ follows from the circumstance that every class of natural numbers has a smallest element. In fact, to a class $A$ of pairs of numbers there exists by the class theorem the subclass of those pairs $\langle m, n\rangle$ such that $\langle m, k\rangle$ does not belong to $A$ for any $k \in n$.

That the axioms $\mathrm{Va}-\mathrm{d}$ hold for $\mathfrak{M}$ follows by applying the following elementary fact (which is a consequence of the theorems on finite sets proved in Part II). If $A$ is a finite class of natural numbers, then there exists the sum $\sum_{k \eta A} 2^{k}$ of the $k^{\text {th }}$ powers of 2 extended over the elements $k$ of $A$. This indeed amounts to stating that every finite class of new sets is represented in the new sense by a set. For instance, the class of the new subsets of a new set $n$ is in the old sense a class of natural numbers not greater than $n$, so it is a finite class and therefore by the theorem just stated it is represented in the new sense by a set; thus Vd holds for $\mathfrak{M}$.

Axiom VII follows by using that every non-empty class of natural numbers has a smallest element and that each element, in the new sense, of a number $m$ is a number smaller than $m$.

So indeed the Ackermann model $\mathfrak{M}$ is found to satisfy all our axioms besides the axiom of infinity.

Now a slight modification of the method used here allows us to construct the models which were announced in Part II.

\footnotetext{
"In the sense of Part I, pp. 70-71; for the proof see Part II, pp. 11-14.
} 
By means of the first, which we shall call $\mathfrak{M}_{1}$ we are to show that the existence of a set ${ }^{87}$ which is its own only element is compatible with the axioms I-III and Va and even with all the axioms $I-V$.

The model $\mathfrak{M}_{1}$ is obtained from $\mathfrak{M}$ by merely interchanging $0 \tilde{\boldsymbol{\epsilon}} n$ and $1 \tilde{\epsilon} n$ in the definition of elementhood between new sets. Thus the definition of $m \tilde{\boldsymbol{\epsilon}} n$ for $m \geq 2$ is the same in $\mathfrak{M}_{1}$ as in $\mathfrak{M}$, but in $\mathfrak{M}_{1}$ we have $1 \in n$ if and only if $n$ is odd, and $0 \tilde{\epsilon} n$ if and only if $n$ is of one of the forms $4 k+2$ or $4 k+3$. According to this a new set is again determined by its elements. Moreover the number which in $\mathfrak{M}_{1}$ is the new set with the elements $m_{1}, \ldots, m_{k}$ is obtained from the number $2^{m_{1}}+\ldots+2^{m_{k}}$ by replacing the exponent 0 , if it occurs, by 1 , and likewise 1 by 0 .

There is now no difficulty in adapting to $\mathfrak{M}_{1}$ the verification of the axioms $\mathrm{I}-\mathrm{V}$ given for the model $\mathfrak{M}$. But axiom VII is not satisfied, since the new set 1 is its only element.

This method of modifying $\mathfrak{M}$ can be sharpened so as to produce models in which the axiom VII is violated in a stronger way. We give two instances.

Let us define $n^{*}$, for a natural number $n$, as follows: if $n$ is odd, then $n^{*}$ is $2^{n}$; if $n=2^{k}$ with $k$ odd, then $n^{*}$ is $k$; in all other cases, $n^{*}$ is $n$. According to this obviously $\left(n^{*}\right)^{*}=n$. Now the element relation $m \tilde{\boldsymbol{\epsilon}} n$ is defined to mean that $m^{*}$ occurs as an exponent in the dyadic expansion of $n$. The condition can also be expressed in this way: we have $m^{*} \tilde{\epsilon} n$, if and only if $m$ occurs as an exponent in the dyadic expansion of $n$. Then again all the axioms $\mathrm{I}-\mathrm{V}$ can be verified to hold. (Indeed the element relation can again be formulated by a constitutive expression, and likewise the relation between $m, n$ and $\langle m, n\rangle$, since the ordered pair $\langle m, n\rangle$ is now $2^{\left(2^{m *}\right)^{*}}$ for $m=n$, and $2^{\left(2^{m *}\right)^{*}}+2^{\left(2^{m *}+2^{n *}\right)^{*}}$ otherwise.) Axiom VII is now violated in such a way that for each odd number $m$ we have $\left(2^{m}\right)=2^{m}$. Thus we have an enumerable class of new sets which are their only elements.

The other instance differs from the foregoing only by the definition of the star-function. Namely we define: for $n$ odd, $n^{*}$ is $2^{n+2}$; for $n=2^{k+2}$ with $k$ odd, $n^{*}$ is $k$; in all other cases, $n^{*}$ is $n$. Here the deviation from axiom VII is such that we have an enumerable class of new sets where the set $a_{k}$, assigned by the enumeration to the number $k$, has $a_{k+1}$ as its element. Indeed we have $2^{2 k+1}=\left(2^{2 k+3}\right)=\left(\left(2^{2 k+5}\right)\right)$, and so on.

Remark. The model $\mathfrak{M}_{1}$ and also the two subsequent models of a set theory not satisfying the axiom VII can be extended to satisfy also the axiom VI (the axiom of infinity), provided this axiom and the axioms $V$ are included in the basic system. Namely we can perform a construction fully parallel to that of the $\Pi$-system with only the following differences: (1) As starting set we take not the null-set but the set of all natural numbers, which exists as a consequence of the axiom VI.

${ }^{37}$ Cf. Part II, p. 9. 
(2) Every finite set of natural numbers which is not itself a natural number is left out.

(3) The element relation between natural numbers is defined in the same way as it is in the model to be extended.

The replacement process (2) has to be repeated at each step of the extension of the system of sets by the successive formations of power sets and sums. In the resulting system of sets, which in the basic system (by the general recursion theorem) is a class $S$, every set, as in the $\Pi$-model, has a degree. For every natural number the degree is null; for other sets it is a successor ordinal.

The classes of the new system are the subclasses of $S$, taken in the basic system. The element relation between sets belonging to $S$ - with the exception of that one newly defined between natural numbers - is the original one, likewise that between sets and classes.

It can be shown that the system of sets and classes so constructed satisfies the axioms I-III, V, VI, and also the axiom IV if this is satisfied in the basis. So from the extended models it follows that, if we can satisfy the axioms I-VI (with or without axiom IV), we can satisfy them in such a way that there exists an infinite set each element of which is its own only element, ${ }^{88}$ and also in such a way that there exists an infinite sequence each member of which has the following member as its element.

Now we come to the other announced model, ${ }^{89}$ which we call $\mathfrak{M}_{2}$. By this we are to show that without $\mathrm{Va}$ it cannot be proved (a) that a transitive set having every transitive proper subset as an element is an ordinal, nor (b) that a set is an ordinal if it satisfies Zermelo's defining conditions for $n$ being an ordinal, which are: $0=n \vee 0 \in n ; a \in n \rightarrow a+(a)=n \vee a+(a) \epsilon n ;$ if $s \subseteq n$, then the sum of the elements of $s$ is represented either by $n$ or by an element of $n$.

The proofs are impossible not only on the basis of I-III and VII, but even if IV and Vc are added. The availability of Vc is particularly agreeable for the consideration of Zermelo's conditions, since by it the third of these conditions becomes simpler in the sense that the representation of the sum of the elements of $s$ by a set holds already in virtue of Vc. Of course Zermelo in setting up his definition presupposed the holding of $\mathrm{Vc}$.

The model $\mathfrak{M}_{2}$ is again number-theoretic. As before, the class relation in it is the ordinary one of a number belonging to a class of numbers, and the element relation $\tilde{\epsilon}$ is again connected with the dyadic expansion of numbers, but in a more complicated way. In order to facilitate its formulation let us take $0 c(k, m)$ as an abbreviation for " $k$ occurs as an exponent in the

${ }^{88}$ This has been recently shown by another method by Ernst Specker in his Habilitationsschrift of 1952 , soon to appear.

${ }^{89}$ Cf. Part II, p. 10, bottom. 
dyadic expansion of $m$ ". Further we use $2 / n$ for " $n$ is even" and $2+n$ for " $n$ is odd". We then have the following definition by cases: $:^{\mathbf{9 0}}$

(1) If $2 / n$, then $m \tilde{\epsilon} n \leftrightarrow 2 / m \& 0 \mathrm{c}\left(\frac{m}{2}+1, n\right)$,

(2) if $2+n$ and $(E x)(x \neq 0 \& 2 / x \& O c(x, n))$, then $m \tilde{\epsilon} n \leftrightarrow O c(m+1, n)$,

(3) if $2+n$ and $(x)(x \neq 0 \& 0 \mathrm{c}(x, n) \rightarrow 2+x)$, then $m \tilde{\epsilon} n \leftrightarrow 2 / m \vee 0 \mathrm{c}(m, n)$.

This definition may be explained as follows. The three cases on $n$ correspond to three kinds of new sets:

(1) those whose transitive closures are finite,

(2) those which themselves are finite whereas their transitive closures are infinite,

(3) those which are infinite.

Concerning the elements of a new set $n$, we see that, if $n$ is of the first kind, its elements are the numbers $2 k-2$ such that $0 \mathrm{c}(k, n)$. If $n$ is of the second kind, then its elements are the predecessors of the exponents $\neq 0$ in its dyadic expansion. If $n$ is of the third kind, then its elements are the even numbers together with the odd exponents in the dyadic expansion of $n$ if there are such exponents.

The idea of this arrangement is that besides "normal sets", which are those finite sets whose transitive closures are finite and which arithmetically (in the basic system) are characterized as the even numbers, we have also the set of all normal sets corresponding to the "old" 1 and all those nonnormal sets which are generated from 0 and the old 1 by the iterated operation of adding an element (already obtained) to a set. One also sees that every finite set of normal elements occurs as a normal element, and every other finite set occurs as an element of second kind, and every set which has as elements all normal sets besides (at most) finitely many other sets occurs as a set of third kind.

Now we proceed to verify the axioms I-IV, Vc and VII for this model $\mathfrak{M}_{2}$.

As to extensionality, one easily sees that sets in $\mathfrak{M}_{2}$ which have the same elements must be of the same kind and then also must be identical.

For the axioms $I I$, we have first that 0 is the empty set in $\mathfrak{M}_{2}$. For axiom II(2), we shall explicitly indicate how to obtain from a number $n$ which is a new set the number $\sigma(n, m)$ which in $\mathfrak{M}_{2}$ is the set arising from $n$ by adding a set $m$, not yet in $n$, as an element. Here we have to distinguish three cases relative to the kind of $n$.

(1) If $n$ is of the first kind, we have to consider two possibilities according as $m$ is even or not. If $m$ is even, then $\sigma(n, m)=n+2^{\frac{m}{2}+1}$. If $m$ is odd, then $\sigma(n, m)=\left(n_{1}+1\right)+2^{m+1}$, where $n_{1}$ results from $n$ by replacing every additive term $2^{k}$ in the dyadic expansion of $n$ by $2^{2 k-1}$; indeed $n$

${ }^{90}$ We use here "↔" instead of "if and only if". 
is of the first kind and $\sigma(n, m)$ has to be of the second kind, so that for the representation of the elements by the exponents we have to make the passage from the first to the second case.

(2) If $n$ is of the second kind, we have $\sigma(n, m)=n+2^{m+1}$, since this number is also of the second kind.

(3) If $n$ is of the third kind, $m$ must be odd and we obviously have $\sigma(n, m)=n+2^{m}$.

In order to define $\sigma(n, m)$ for arbitrary numbers, we need only take $\sigma(n, m)=n$ for the case that the new set $m$ is in the new set $n$. - By means of $\sigma(n, m)$ we can express the function which is the number-theoretic representation of the ordered pair $\langle a, b\rangle$. Indeed, we have $\langle a, a\rangle=$ $\sigma(0, \sigma(0, a))$, and for $a \neq b,\langle a, b\rangle=\sigma(\sigma(0, \sigma(0, a)), \sigma(\sigma(0, a), b))$.

For the axioms III and IV the verification can be made in the same way as for the model $\mathfrak{M}$; in particular it is necessary to take into account here that in $\mathfrak{M}_{2}$ the element relation and also the relation $\langle a, b\rangle=c$ can be formulated by a constitutive expression. As a consequence of the axioms III the class theorem holds again in $\mathfrak{M}_{2}$.

In order to see that $V_{c}$ holds in $\mathfrak{M}_{2}$, it is sufficient, on account of our previous statements about the different kinds of new sets, to observe that the sum of the elements of any new set is either a finite set of new sets or has as elements all normal sets besides at most finitely many non-normal sets.

That axiom VII is also satisfied results as follows. Let $A$ be any nonempty class of $\mathfrak{M}_{2}$. Two possibilities are to be considered. (a) $A$ has at least one even number as element. Let $n$ be the smallest among them. Then $n$ has no element in common with $A$. For, if $k \tilde{\epsilon} n$, then $2 / k$ and $2^{\frac{k}{2}+1} \leq n$, hence $\frac{k}{2}<\frac{n}{2}, k<n$, and thus $k$ cannot belong to $A$. (b) $A$ has only odd numbers as elements. Among these there is a smallest one $n$. Then again $n$ has no element in common with $A$. Indeed such a common element $k$ would be odd, and since $n$ is of the second or third kind, we should have for an odd element $k$ of $n$ that $2^{k} \leqq n$ or even $2^{k+1} \leq n$, and thus $k<n$.

But axiom $\mathrm{Va}$ obviously is not satisfied. Indeed an infinite subclass of 1 or of any new set of the third kind is represented by a set only if it has all even numbers as elements.

Similarly we find that neither $\mathrm{Vb}$ nor $\mathrm{Vd}$ holds in $\mathfrak{M}_{2}$. For $\mathrm{Vb}$ this follows by considering any one-to-one correspondence of the new set 1 to a proper infinite subclass. As to $\mathrm{Vd}$, let us show that the new set 3 is a counterexample. Indeed $3=2^{\circ}+2^{1}$; thus 3 is of the third kind, and its elements are 1 and the even numbers. Now the set of the second kind $1+2^{2}+2^{2 k+1}$ whose elements are 1 and $2 k$ is a subset of 3 . Since $k$ is arbitrary, there are infinitely many odd numbers (non-normal sets) which are subsets of 3 , and thus are 
elements of the class of all subsets of 3 . This class therefore cannot be represented by a set, because a new set of any kind has only finitely many odd numbers as elements.

Now we are able to prove our aforementioned assertion that on the basis of the axioms I-IV, Vc and VII : (a) the conditions on a set that it be transitive and that every proper transitive subset be an element of it are not sufficient for a characterization of ordinals, and (b) likewise the conditions constituting Zermelo's definition of an ordinal formulated in Part II are not sufficient for the same purpose.

This indeed follows from the consideration of the new set 1 . In $\mathfrak{M}_{2}, 1$ is not an ordinal, as can be seen as follows: $4 \bar{\epsilon} 1,2$ is the only element of 4 , and $0 \tilde{\epsilon} 2$, therefore 4 is not transitive and thus is not an ordinal; so neither is 1 an ordinal. On the other hand, 1 satisfies the conditions that it be transitive and every proper subset of 1 be an element of 1 . For, every element of 1 is a normal set, and each element of a normal set is again such a set; and further every proper subset of 1 must be of the first kind, and therefore is an element of 1.

But also the Zermelo conditions for a set being an ordinal are satisfied by 1 . In fact $0 \tilde{\epsilon} 1$; if $n \tilde{\epsilon} 1$, then $\sigma(n, n) \tilde{\epsilon} 1$; and if $s$ is a subset (in the new sense) of 1 , then the new sum of the elements of $s$ is either 1 , or is a normal set and then is an element of 1 .

19. Reduction of the set-theoretic basis, including classes, to a number-theoretic frame. Our construction of the models $\mathfrak{M}, \mathfrak{M}_{1}, \mathfrak{M}_{2}$ has been performed in the frame of the axiomatic system consisting of the axioms I-III, VII or else I-III, Va. This basis is wider than that frame of set theory whose consistency, as shown by Ackermann's reasoning (l.c.), results as a consequence of the constructive proofs of the consistency of number theory given by Gentzen, Kalmár, Ackermann, Lorenzen, Schütte and Stenius ${ }^{91}$. Indeed we have here the strengthening by the addition of the classes; however this strengthening is only of a restricted character in so far as we do not apply the concept of class in an impredicative way, so that the introduction of classes serves mainly to embody a part of metamathematics in the axiomatic system itself. This fact suggests the conjecture that it will be possible also for the frame considered to reduce the consistency proof to that of number-theory. A natural idea for this is to represent the classes by Gödel numbers, or more exactly by those of number-theoretic expressions corresponding to the constitutive expressions for them. But here a difficulty arises initially with regard to the $\eta$-relation (of a set belonging to a class). We should tend to express this relation by

${ }^{91}$ E. Stenius; Das Interpretationsproblem der formalisierten Zahlentheorie und ihre formale Widerspruchsfreiheit, Acta Academiae Aboensis (Math. et Phys.) vol. 18. no. 3, Åbo Akademi, Åbo 1952, 102 pp. 
that of a set satisfying a constitutive expression. But this relation, as results from Tarski's theory of the truth concept, transgresses the formal system of number theory. On the other hand, if we replace the satisfaction (Erfüllung) of a constitutive expression by a provable satisfaction, then the holding or not holding of an $\eta$-relation would no longer be exhaustive alternatives.

This dilemma can be overcome by the procedure of Ilse Novak ${ }^{92}$ which is based essentially on the method developed by Leon Henkin ${ }^{93}$. We will not presuppose familiarity with these methods, but explain their application to our case independently, making use of a simplification applied by Gisbert Hasenjäger ${ }^{94}$ to the method of Henkin.

What we want to get is a model of our basic system, i.e. of the axioms I-III and VII, set up in the frame of the number-theoretic formal system $Z$ of Grundlagen der Mathematik with certain additions to be mentioned presently. In making these additions we shall use an accessory result of the cited constructive consistency proofs for number theory, namely that the consistency of the system $Z$ is preserved by the following kinds of additions: first addition of a finite number of primitive recursive definitions, second addition of a verifiable recursive formula as an axiom. Obviously also the consistency is not disturbed by adding numbered individual symbols $u_{0}, u_{1}, u_{2}, \ldots$

In order now to prepare the Lindenbaum completion process we first assume a fixed Gödel numbering of the expressions of $Z$ with the added functors and individual symbols included. The next step consists in adding as axioms the formulas $(E x) \mathfrak{A}(x) \rightarrow \mathfrak{A}\left(u_{j}\right)$ where $(E x) \mathfrak{A}(x)$ is any closed formula of the indicated form and $j$ is the Gödel number of this formula. No contradiction can arise by the addition of these formulas to the former consistent system. For otherwise already finitely many such formulas $(E x) \mathfrak{A}_{\nu}(x) \rightarrow$ $\mathfrak{A}_{p}\left(u_{j_{p}}\right)(p=1,2, \ldots \mathfrak{x})$ would yield a contradiction, and if $j_{\mathfrak{x}}$ is the greatest of the $j_{p}$, then by the deduction theorem it would follow that from the formulas $(E x) \mathfrak{A}_{p}(x) \rightarrow \mathfrak{A}_{p}\left(u_{j_{p}}\right)$ with $p<\mathfrak{x}$ (on the basis of our numbertheoretic system) the negation of $(E x) \mathfrak{A}_{\mathfrak{r}}(x) \rightarrow \mathfrak{U}_{\mathfrak{r}}\left(u_{j_{\mathfrak{r}}}\right)$ and thus the formulas

${ }_{92}$ I. Novak, A construction for models of consistent systems, Fundamenta mathematicae, vol. 37 (1950), pp. 87-110.

${ }_{93} \mathrm{~L}$. Henkin, The completeness of the first-order functional calculuts, this Journal, vol. 14 (1949), pp. 159-166. An essential step in Henkin's procedure is the construction of a complete set of formulas from any consistent set of formulas, on some logical basis. This method goes back to A. Lindenbaum; see A. Tarski, Fundamentale Begriffe der Methodologie der deduktiven Wissenschaften I, Monatshefte für Mathematik und Physik, vol. 37 (1930), Satz I. 56, p. 394. We shall therefore refer to this method, which (in the form in which we have to use it) will be explained, as the "Lindenbaum completion process".

9.1 G. Hasenjäger, Eine Bemerkung zu Henkin's Beweis für die Vollständigkeit des Prädikatenkalküls der ersten Stufe, this Journal, vol. 18 (1953), pp. 42-48. 
$(E x) \mathfrak{A}_{\mathfrak{r}}(x)$ and $\mathfrak{A}_{\mathfrak{r}}\left(u_{j_{\mathfrak{r}}}\right)$ could be derived. But then also, since $u_{j_{\mathfrak{r}}}$ cannot occur within $\mathfrak{A}_{p}(x)$ for $p<\mathfrak{x}, \mathfrak{A}(v)$ would be derivable for any free variable $v$ and thus also $(x) \mathfrak{A}_{\mathfrak{r}}(x)$. So already the first $r-1$ of the formulas $(E x) \mathfrak{A}_{p}(x) \rightarrow$ $\mathfrak{A}_{p}\left(u_{j_{p}}\right)$ would lead to a contradiction. However this possibility can be excluded by assuming from the beginning that the index $\mathfrak{x}$ has been chosen as the minimal one for which a contradiction arises from formulas $(E x) \mathfrak{A}(x) \rightarrow$ $\mathfrak{U}\left(u_{j}\right)$ (out of our series) with $j \leq r$.

Let us denote by $Z_{\mathrm{I}}$ the formal system which we obtain by adding to the system $Z$ the said recursive definitions - we introduce as many of them as are sufficient for performing the Gödel arithmetization of syntax and the elementary theory of dyadic expansion (as used in our model) -, the individual symbols $u_{\mathfrak{t}}$, and the accessory axioms $(E x) \mathfrak{R}(x) \rightarrow \mathfrak{A}\left(u_{j}\right)$. As we have seen, $Z_{\mathrm{I}}$ like $Z$ is a consistent system.-

Now we are to perform Lindenbaum's completion process; however it will be sufficient to do this only implicitly by defining a number-theoretic predicate formulating the property of a number $\mathfrak{f}$ of being the Gödel number of a closed formula which is derivable in the completed system. This predicate corresponds fully to $I$. Novak's predicate $T$, and we shall denote it by the same letter.

Let us first give the definition of $T(k)$ in an informal way, and afterwards indicate how to formalize it in the frame of $Z_{\mathrm{I}}$. We begin by introducing the concept of an extension set of $Z_{\mathrm{I}}$. By an extension set of $Z_{\mathrm{I}}$ we understand a finite set of closed formulas of $Z_{\mathrm{I}}$ which, if added to $Z_{\mathrm{I}}$, do not yield a contradiction. An extension set can be indicated by the set of the Gödel numbers of its formulas, and this set can be represented by that number in whose dyadic exparsion those Gödel numbers are the exponents which occur. Thus a number $n$ will be said to represent an extension set of $Z_{I}$, if the exponents in the dyadic expansion of $\mathfrak{n}$ are the Gödel numbers of closed formulas which added to $Z_{\mathrm{I}}$ as axioms preserve consistency. One extension set deviates from another, if neither of them is a subset of the other. - Of two extension sets deviating from each other, that one is called anterior which contains the formula with the smallest of those Gödel numbers occurring for one of them but not for both. Now $T(k)$ is the following number predicate: " $k$ is the Gödel-number of a formula of some extension set which is anterior to every extension set that deviates from it".

For the formalizing we make use of the primitive recursive relation $\tilde{\epsilon}$ of our model MY. Further we denote by "Es $(n)$ " the predicate " $n$ represents an extension set of $Z_{\mathrm{I}}$ ". The above definition of this predicate obviously can be formalized by means of the Gödel arithmetization of the syntax of $Z_{\mathrm{I}}$. The concepts "deviating" and "anterior" are formalized by the explicit definitions

$\operatorname{DV}(k, l) \leftrightarrow(E x)(x \tilde{\boldsymbol{\epsilon}} k \& \overline{x \tilde{\epsilon} l)} \&(E x) \overline{(x \tilde{\boldsymbol{\epsilon}} k} \& x \tilde{\epsilon} l)$,

Ant $(k, l) \leftrightarrow(E x)[(y)(y<x . \rightarrow y \tilde{\epsilon} k \leftrightarrow y \tilde{\epsilon} l) \& x \tilde{\epsilon} k \& \bar{x} \tilde{\boldsymbol{\epsilon}} l]$. 
Now the formal definition of $T(k)$ is

$T(k) \leftrightarrow(E x)[\operatorname{Es}(x) \&(y)(\operatorname{Es}(y) \& \operatorname{Dv}(x, y) \rightarrow \operatorname{Ant}(x, y)) \& k \tilde{\epsilon} x]$.

This definition of $T(k)$ can be shown by means of complete induction to be equivalent to the following recursive condition: $T(k)$, if and only if $k$ is the Gödel-number of a closed formula which on the basis of $Z_{\mathrm{I}}$ is compatible with all those closed formulas whose Gödel-numbers are lower than $k$ and have the property $T$. Using the consistency of $Z_{\mathrm{I}}$, which is formally expressible by a verifiable primitive recursive formula whose addition to $Z_{\mathrm{I}}$ as an axiom preserves the consistency, we can prove that there are infinitely many numbers $k$ such that $T(k)$. By calling a formula whose Gödel number has the property $T$ a $T$-formula, as we shall do in the following, we can express the last statement by saying that there are infinitely many $T$-formulas.

Also it can be seen that every closed formula provable in $Z_{\mathrm{I}}$ is a $T$ formula, and that every closed formula derivable by means of $Z_{\mathrm{I}}$ from $T$-formulas is again a $T$-formula.

Further with regard to the logical operations we have the statements: (1) $\overline{\mathfrak{A}}$ is a $T$-formula, if and only if $\mathfrak{A}$ is not a $T$-formula. (2) $\mathfrak{A} \& \mathfrak{B}$ is a $T$-formula, if and only if $\mathfrak{A}$ and $\mathfrak{B}$ are $T$-formulas. (3) $(E x) \mathfrak{A}(x)$ is a $T$ formula, if and only if there is a number $j$ such that $\mathfrak{A}\left(u t_{j}\right)$ is a $T$-formula. We can say even more, namely that $(E x) \mathfrak{A}(x)$ is a $T$-formula, if and only if, when $\mathfrak{r}$ is its Gödel-number, $\mathfrak{A}\left(u_{\mathfrak{r}}\right)$ is a $T$-formula. In order to express formally in $Z_{I}$ the last condition we have to apply the primitive recursive function $\mathfrak{U}(r)$ (which can be composed out of the recursive functions introduced in $Z_{\mathrm{I}}$ ) whose value for a definite value $r$ of $r$ is the Gödel-number of $u_{\mathfrak{r}}$. Statements corresponding to (1)-(3) hold for the other logical connectives in virtue of their expressibility by means of the three mentioned. For illustration let us consider the following example, for which we use the original form of Gödel numbering of formulas. Let $\mathfrak{y}, \mathfrak{s}, \mathfrak{p}, \mathfrak{n}$ be the numbers (Zeichennummern) of the symbols $=,+\cdot, 0$, and let be the number of the formula $(x)\left(x \neq 0 \rightarrow(E y)(E z)\left(x \cdot y=u_{128}+z\right)\right)$. Then we have $T(\mathfrak{f}) \leftrightarrow(x)\left[\bar{T}\left(2^{\mathfrak{u}(x)} \cdot 3^{\mathfrak{h}} \cdot 5^{\mathfrak{n}}\right) \rightarrow(E y)(E z) T\left(2^{\mathfrak{u}(x)} \cdot 3^{\mathfrak{p}} \cdot 5^{\mathfrak{u}(y)} \cdot 7^{\mathfrak{y}} \cdot 11^{\mathfrak{u}(128)} \cdot 13^{\mathfrak{a}} \cdot 17^{\mathfrak{u}(z)}\right)\right]$.

We are now ready to set up our number-theoretic model of the system of the axioms I-III and VII. We do this by describing the model in the frame of the metarnathematics of $Z_{\mathrm{I}}$ including the theory of the predicate $T$. The whole reasoning can be formalized in the frame of $Z_{I}$ with the addition of the consistency formula as an axiom. As this formal frame is shown to be consistent by Gentzen's and the other constructive proofs, from the same proofs it will follow that our system I-III and VII is also consistent, and so the consistency of the system of our axioms $I-V$ and VII proved by the model $\mathfrak{M}$ and the independencies proved by means of the models $\mathfrak{M}_{1}, \mathfrak{M}_{2}$ hold also as a consequence of the constructive consistency proofs. 
In describing the metamathematical model we do not enter into all the details of the number-theoretic formalization, the method of which is indeed well known. We shall apply the number-theoretic notations, where it seems useful for clarity.

As sets we take the individual-symbols $u_{0}, u_{1} \ldots$ Equality between $u_{i}$ and $u_{\mathfrak{t}}$ is not to be understood as simple identity, but rather we define $u_{i}$ to be equal to $u_{i}$ if the equation $u_{\mathfrak{i}}=u_{\mathfrak{i}}$ is a $T$-formula, or what is the same, if $T\left(2^{\mathfrak{u}(i)} \cdot 3^{\mathfrak{h}} \cdot 5^{\mathfrak{u}(\mathfrak{l})}\right)$. As we see, this relation, briefly " $i=k_{T}$ ", is not directly a predicate of $u_{i}$ and $u_{k}$ but rather of $i$ and $k$; yet we can regard it as referring to the symbols $u_{i}$, since these are assigned in a one-to-one way to their indices.

In a like indirect way we define the element-relation between $u_{i}$ and $u_{\mathfrak{t}}$ by a number-theoretic predicate " $i \tilde{\epsilon} k$ " which holds for $\dot{i}$ and $\mathfrak{t}$ if and only if the result of substituting $u_{i}$ for $a$ and $u_{i}$ for $b$ in the number-theoretic formula $\mathbb{E}(a, b)$ which expresses the relation $a \tilde{\epsilon} b$ (i.e. " $a$ occurs as exponent in the dyadic expansion of $\left.b^{\prime \prime}\right)$ is a $T$-formula.

As classes we take the propositional expressions of our system $Z_{\mathrm{I}}$, with a distinguished variable $c$ (Nennvariable) as their only free variable. These expressions of course have Gödel numbers and therefore can be enumerated as $\mathfrak{P}_{0}(c), \mathfrak{P}_{1}(c), \ldots$ It may be noted that the Gödel number of $\mathfrak{P}_{\mathfrak{l}}(c)$ depends primitive recursively on $\mathfrak{k}$, since we can give a primitive recursive majorant for the sequence of the Gödel numbers of the $\mathfrak{B}_{\mathfrak{t}}(c)(\mathfrak{k}=0,1, \ldots)$.

As the predicate of a set belonging to a class we take the relation " $i \eta_{T} k$ " which holds for $\mathfrak{i}$ and $\mathfrak{i}$ if the formula $\mathfrak{B}_{\mathfrak{t}}\left(u_{\mathfrak{i}}\right)$ is a $T$-formula.

Finally we define equality between $\mathfrak{F}_{\mathfrak{i}}(c)$ and $\mathfrak{F}_{\mathfrak{f}}(c)$ to mean that the formula $(x)\left(\mathfrak{P}_{\mathfrak{i}}(x) \leftrightarrow \mathfrak{P}_{\mathfrak{i}}(x)\right)$ is a $T$-formula.

We can now show that the axioms I-III and VII are satisfied. For these verifications we have to make repeated use of the fact, already stated, that the predicate "being a $T$-formula" commutes with the logical connectives (including the quantifiers).

From this it follows that first the (preliminary) axioms of equality and the axioms of extensionality are satisfied. Let us illustrate this by the case of the extensionality axiom for sets. What we have to show is that, if for

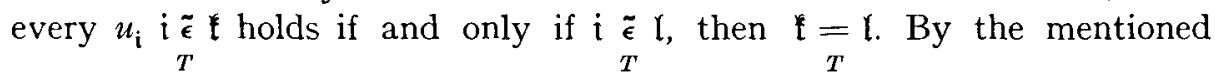
commutativity of the predicate "being a $T$-formula", this comes to the same as:

if

is a $T$-formula, then

is a $T$-formula. Now in $Z_{\mathrm{I}}$ we can prove

$$
(x)\left(\mathbb{E}\left(x, u_{\mathfrak{l}}\right) \leftrightarrow \mathbb{E}\left(x, u_{\mathfrak{l}}\right)\right) \rightarrow u_{\mathfrak{l}}=u_{\mathfrak{l}} .
$$


Thus this formula - let us abbreviate it $\mathfrak{U} \rightarrow \mathfrak{B}$ - is a $T$-formula, and so, if $\mathfrak{A}$ is a $T$-formula, $\mathfrak{B}$ is a $T$-formula. But just this had to be shown.

As to the axiom II 1, we have that the formula $(E x)(z) \overline{(E(z, x)})$ is provable in $Z_{\mathrm{I}}$ and thus is a $T$-formula. Let $n_{0}$ be the Gödel number of this formula; then also $(z)\left(\overline{\mathbb{E}\left(z, u_{\mathfrak{n}_{0}}\right)}\right)$ is a $T$-formula. Thus $u_{\mathfrak{n}_{0}}$ has the rôle of the null set.

For the verification of II 2 , we have to prove

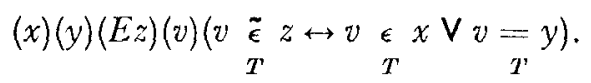

By the commutativity of the predicate "being a $T$-formula" this is equivalent to stating that the formula

$$
(x)(y)(E z)(v)(\mathfrak{E}(v, z) \leftrightarrow \mathfrak{E}(v, x) \vee v=y),
$$

is a $T$-formula. However this results from the fact that this formula is provable in $Z_{\mathrm{r}}$.

For the axioms III the task of verification amounts to showing that in each case the class stated in the axiom to exist is characterized in our model by the condition on the elements $u_{\mathfrak{i}}$ that $\underset{i}{i} \eta_{T}$ holds for a certain propositional expression $\mathfrak{P}_{\mathfrak{f}}(c)$. For this a difficulty might seem to arise from the circumstance that in the defining conditions for the classes certain classes occur as parameters. However, since in our model the classes are themselves predicates (i.e. propositional expressions), this is no hindrance. Besides we have everywhere to make use again of the commutativity of the predicate "being a $T$-formula".

Let us illustrate the method of verification for the case of the axiom c(1), which we can satisfy even in the strengthened form (as it occurs in Gödel's monograph ${ }^{95}$ ): "For every class $A$ there exists a class whose elements are those sets which are the first members of ordered pairs belonging to $A$." In order to express that this assertion holds for our model, we have to translate the condition " $a$ is the first member of an ordered pair belonging to a given class $A$ ", i.e.

$$
\begin{aligned}
(E t)\{(E x)(E y)(E z)(u)(v)(w)[(u \in x \leftrightarrow u=a) \&(v \in z \leftrightarrow v=a \vee v=y) \& \\
\&(w \in t \leftrightarrow w=x \vee w=z)] \& \operatorname{t} \eta A\},
\end{aligned}
$$

into a condition for a $u_{\mathrm{i}}$ with respect to a propositional expression $\mathfrak{B}_{\mathrm{t}}(c)$. This is to be done by the following steps: First we replace the set variables by number variables (ranging over the indices of the $u$-symbols), but we need not change the letters. Further we change every " $\epsilon$ " into " $\tilde{\epsilon}$ ", every "=" into " $="$ "; and finally we replace the conjunctive member $t \eta A$ by

${ }^{95} \mathrm{~K}$. Gödel, The consistency of the continuum hypothesis, Annals of mathematics studies, No. 3 (1940), axiom B5. 
$t \underset{T}{\eta}$. Now what we have to recognize is that the resulting formula

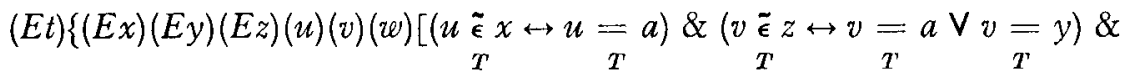

$$
\begin{aligned}
& (w \underset{T}{\tilde{\epsilon}} t \leftrightarrow \underset{T}{=} x \vee \underset{T}{=}=z)] \underset{T}{\&} t \underset{T}{\eta} k\},
\end{aligned}
$$

with the variable $a$ replaced by the numeral $i$ and $k$ by the index $f$ of the propositional expression $\mathfrak{B}_{\mathfrak{p}}(c)$ to be substituted for $A$, expresses that, for a certain propositional expression $\mathfrak{P}_{\mathfrak{r}}(c)$, the formula $\mathfrak{P}_{\mathfrak{r}}\left(u_{\mathfrak{i}}\right)$ is a $T$-formula. But it is easy to find a $\mathfrak{P}_{\mathbf{r}}(c)$ of this kind. Indeed by virtue of the already repeatedly applied commutativity of the predicate "being a $T$-formula", we get it from our first formula by letting the variables $t, x, y, z, u, v, w$ range over the $u$-symbols instead of over sets, and replacing $a$ by $c$, and every expression $\mathfrak{a} \in \mathfrak{b}$ by the corresponding $\mathfrak{E}(\mathfrak{a}, \mathfrak{b})$, and the expression $t \eta A$ by $\mathfrak{P}_{\mathfrak{r}}(t)$.

The method used in this instance applies correspondingly to all the axioms III.

Now it only remains to state that also axiom VII is fulfilled in our model. Its assertion for this model is that for any propositional expression $\mathfrak{P}_{\mathfrak{t}}(c)$ such that there is a $u_{\mathfrak{i}}$ for which $\mathfrak{P}_{\mathfrak{i}}\left(u_{\mathfrak{t}}\right)$ is a $T$-formula, there is a $u_{\mathfrak{i}}$ for which $\mathfrak{P}_{\mathfrak{l}}\left(u_{\mathrm{j}}\right)$ is a $T$-formula, whereas there is no $u_{\mathfrak{l}}$ such that $\mathbb{E}\left(u_{\mathfrak{l}}, u_{\mathrm{j}}\right)$ and $\mathfrak{P}_{\mathfrak{l}}\left(u_{\mathfrak{l}}\right)$ are both $T$-formulas. Or formally expressed,

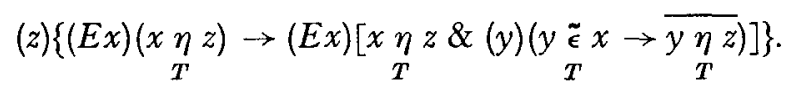

The proof of this can be sketched as follows. In $Z_{\mathrm{I}}$ we can prove

$$
\mathfrak{P}_{\mathfrak{l}}\left(u_{\mathrm{i}}\right) \rightarrow(E x)\left[\mathfrak{P}_{\mathfrak{l}}(x) \&(y)\left(y<x \rightarrow \overline{\mathfrak{P}_{\mathfrak{l}}(y)}\right]\right.
$$

where $y<x$ stands for $(E z)(z \neq 0 \& y+z=x)$. Further we have in $Z_{\mathrm{I}}$

$$
(E x)\left[\mathfrak{P}_{\mathfrak{l}}(x) \&(y)\left(y<x \rightarrow \overline{\mathfrak{F}_{\mathfrak{l}}(y)}\right] \rightarrow \mathfrak{P}_{\mathfrak{l}}\left(u_{\mathfrak{m}}\right) \&(y)\left(y<y_{\mathfrak{m}} \rightarrow \overline{\mathfrak{F}_{\mathfrak{l}}(y)}\right)\right.
$$

where $\mathfrak{m}$ is the Gödel number of the formula standing in the antecedent. Thirdly in $Z_{\mathrm{I}}$ we have $(y)(\mathscr{E}(y, u) \rightarrow y<u)$. Combining the three formulas, we obtain as a provable formula

$$
\mathfrak{P}_{\mathfrak{l}}\left(u_{\mathfrak{i}}\right) \rightarrow \mathfrak{F}_{\mathfrak{l}}\left(u_{\mathfrak{m}}\right) \&(y)\left(\mathbb{E}\left(y, u_{\mathfrak{m}}\right) \rightarrow \overline{\mathfrak{F}_{\mathfrak{r}}(y)}\right) .
$$

From this provability it follows that, if $\mathfrak{P}_{\mathfrak{f}}\left(u_{\mathfrak{i}}\right)$ is a $T$-formula, then the consequent is also; and by virtue of the often mentioned commutativity of $T$, we get the assertion to be proved.

So now we have shown that all the axioms I-III and VII hold for our metamathematical model. By this, as already mentioned, the consistency of the system of these axioms is established in a constructive sense. 
Remark. It may be noticed that it is possible also to show that the axioms IV and $\mathrm{V}$ are likewise satisfied for our model. But for our purpose this statement is not needed, since indeed the model $\mathfrak{M}$ can be established on the basis of the axioms I-III and VII.

20. Elimination of one of the axioms III. Among the axioms III one, as we found, is redundant, namely $b(1)$. We give here its derivation from the other axioms III (without a(1)) and the extensionality axiom I(1) (using also some of the equality axioms.)

The assertion of $b(1)$ is that there exists the class $A$ of all unit sets. We have $A=B-\Gamma$ where $B$ is the class of those sets which have at least one element, and $\Gamma$ the class of the sets with at least two different elements.

(1) We first observe that on the assumption of the existence of $B$ and $\Gamma$ also $B-\Gamma$ exists by $a(2), a(3)$; indeed it is the intersection of $B$ with the complementary class of $\Gamma$. Furthermore the existence of $B$ is immediate from $b(2), c(2)$ and $c(1)$. For, if $E$ is the class required to exist by $b(2)$, then $B$ is the converse domain of $E$. So it will be sufficient to prove the existence of $\Gamma$.

(2) A set $c$ is an element of $\Gamma$, if and only if there exists a triplet $\langle\langle a, b\rangle, c\rangle$ such that $a \in c, b \in c$ and $a \neq b$. If $\Delta$ is the class of these triplets, then $\Gamma$ is the converse domain of $\Delta$. Thus the proof of the existence of $\Gamma$ reduces to the proof of the existence of $\Delta$. Now $\Delta$ can be characterized as an intersection of three classes of triplets $\langle\langle a, b\rangle, c\rangle:(1)$ those with arbitrary $a$ and $b \in c$ (class $\Delta_{1}$ ), (2) those with arbitrary $b$ and $a \in c$ (class $\Delta_{2}$ ), (3) those with arbitrary $c$ and $a \neq b$ (class $\Delta_{3}$ ). The existence of these three classes, under the provisional assumption of the existence of the class $\Theta$ of all pairs $\langle a, b\rangle$ with $a \neq b$, can be shown as follows: $\Delta_{1}$ results by coupling to left (c(3)) from the converse class (c(2)) of the class of triplets $\langle\langle b, c\rangle, a\rangle$ with $\langle b, c\rangle \eta \mathrm{E}$, which exists by $\mathrm{b}(3) . \Delta_{2}$ results from the class of triplets $\langle\langle c, a\rangle, b\rangle$, where $\langle c, a\rangle$ belongs to the converse class of $E$, which class of triplets exists by $\mathrm{b}(2), \mathrm{c}(2), \mathrm{b}(3)$; the passage from this class of triplets to $\Delta_{2}$ is by applying successively $\mathrm{c}(2), \mathrm{c}(3), \mathrm{c}(2), \mathrm{c}(3) . \Delta_{3}$ exists by $\mathrm{b}(3)$ as the class of pairs whose first members belong to $\Theta$.

(3) So now it remains only to prove the existence of $\Theta$. By the axiom I(1) and some of the equality axioms, this class can be characterized as the class of the pairs $\langle a, b\rangle$ for which there exists a set $c$ such that either $c \epsilon a \& c \notin b$ or $c \notin a \& c \epsilon b$. Thus it is the sum of the classes of pairs $\langle a, b\rangle$ with a $c$ existing of the one kind or the other, respectively. Both these classes exist according to the composition lemma, applied for one to the converse class of $E$ and to $\Phi-E$, where $\Phi$ is the class of all pairs, and for the other to the converse class of $\Phi-\mathrm{E}$ and to $\mathrm{E}$. Note that the existence of $\Phi$ follows by applying $b(3)$ to the class of all sets, which exists as the sum of the class $\mathrm{E}$ and its complement, also that the sum of any two classes is 
the complement of the intersection of their complements, and finally that the composition lemma has been derived from the axioms $a(3), b(3), c(1)$, $c(2), c(3) \cdot{ }^{96}$ - So indeed $b(1)$ can be spared. ${ }^{97}$

We add here the following remark: The axiom $b(1)$ was especially used in Part I to prove the existence of the class of pairs $\langle a, b\rangle$ such that $a=b$; and there we observed that this derivation depended on our special definition of the ordered pair. Now we see that the existence of this class of pairs follows directly and without applying a definition of the ordered pair. Indeed the class in question is nothing else than $\Phi-\Theta$.

Remark. We showed how the models $\Pi_{0}, \Pi_{1}, \Pi_{2}$ can be constructed on a narrower axiomatic basis, in particular avoiding the use of the axiom whose independence is to be proved.

On this point Firestone in his review of Part VI ${ }^{98}$ made the observation that generally, if we have a model, based on all the axioms of a system $\mathfrak{S}$, for the independence of a particular axiom $\mathfrak{A}$ from the set $\mathfrak{S}_{0}$ of the other axioms, then this independence follows under the condition of the consistency of $\mathfrak{S}_{0}$. For, on the assumption that the axiom $\mathfrak{A}$ is derivable from $\mathfrak{S}_{0}$, we should be able to construct our model also on the basis of $\mathfrak{S}_{0}$. In this model we should have the negation $\overline{\mathfrak{A}}$ of $\mathfrak{A}$, and on the other hand, since all axioms of $\mathfrak{S}_{0}$ are satisfied in the model and these (as is assumed) entail $\mathfrak{A}$, we should likewise have $\mathfrak{A}$. So a contradiction would follow from $\mathfrak{S}_{0}$.

From the point of view of deducibility this reasoning is of course sufficient. But we are interested not only in the statements of independence resulting from certain models, but also in the models themselves and in the method of their construction. In this respect the above indirect argument certainly cannot replace the second direct constructions of $\Pi_{0}, \Pi_{1}, \Pi_{2}$. Moreover we have there been able not only to avoid the use of the axiom to be proved independent, but also to obtain some further reductions (e.g. Vc is not used in $\Pi_{0}, \Pi_{1}, \Pi_{2}$ ).

Concerning our statement that Zermelo's axioms are satisfied by the system of $\Pi^{*}$-sets, the reviewer raised the question whether this agrees with

\footnotetext{
${ }_{96}$ This JouRNAL, vol. 2 (1937), pp. 75-76.

97 With regard to a remark made by Gödel (l.c., footnote 94, p. 7), it may be noticed that as a consequence of our preceding reasoning Gödel's axioms B7 and B8 are derivable from the axioms III without using $b(1)$.

In fact Gödel's axioms B7 and B8 stand not only for our axiom III c(3), but also for $\mathrm{c}(2)$, as results from the derivability of B6 from B4, B5 and B8 stated by A. A. Markov in On the dependence of axiom B6 on the other axioms of the Bernays-Gödel system, Izvéstiyá Akadémii Nauk. SSSR, ser. mat., vol. 12 (1948), pp. 569-570; cf. Mathematical reviews, vol. 10 (1949), p. 421. On the other hand, it may be observed that upon assuming B6, which is the same as our c(2), Gödel's B7 is equivalent to our $\mathrm{c}(3)$; thus it follows that $\mathrm{B} 8$ is provable from $\mathrm{B} 1-\mathrm{B} 7$.
}

98 This Journal, vol. 13 (1948), pp. 220-221. 
Zermelo's assumption of "Urelemente" different from the null-set. From Zermelo's paper Über Grenzzahlen und Mengenbereiche ${ }^{99}$ it appears however that Zermelo included the case in which the empty set is the only "Urelement”. In fact he speaks of an „Einheitsbereich" as a possible kind of a model, although he intended that the axiom system for set theory in view of its applications should not exclude models with more "Urelemente".

ZURICH

99 Fundamenta mathematicae, vol, 16 (1930), pp. 29-47, especially pp. 37-38. 